\title{
Complex scenarios with competing factors
}

\author{
A conception paper applied to the COVID-19 case
}

\author{
Mauricio Pazini Brandão
}

Received: 18 July 2020 / Accepted: 9 March 2021 / Published online: 21 April 2021

(C) The Author(s), under exclusive licence to Springer Nature B.V. 2021

\begin{abstract}
A theory to analyze complex scenarios facing threats with competing factors and limited resources has been introduced. The scenarios are modeled as closed systems. Hamilton's principle of stationary action is used to conceive a theory in which competing factors dispute available resources to minimize undesirable outcomes. The result indicates that the minimum response is obtained by a combination of the competing factors weighted by their corresponding criticalities. The theory has been applied to the COVID19 pandemic with two competing factors: Health and Economy. As main result, to minimize the total number of deaths, the recommendation is to balance the emphasis on both factors. This implies to give more emphasis to the economic factor, by avoiding restrict interventions like lockdowns and business closures. The model may evolve from a qualitative to a quantitative status, allowing for computational simulations aimed at validations and forecasting. As such, this approach may become a useful tool for strategic decision-making regarding resources allocations to reduce guessing in scenarios full of uncertainties.
\end{abstract}

Keywords Hamilton's principle - Optimization · Resources allocation - Strategic decision-making · Macroeconomics · Epidemics · COVID-19

M. P. Brandão ( $\square)$

Secretariat for Strategic Studies (SAE-PR), Brazilian Republic Presidency, Palácio do Planalto, Brasília, DF 70150-900, Brazil

e-mail: pazinibrandao@gmail.com

\section{A hypothetical scenario or the rules of the game}

No matter how complex a situation might be, there might be a way of considering it from a simpler perspective. In this paper we introduce an approach to treat a general problem with an elementary yet powerful tool inspired in Nature.

Let us imagine some sort of domain (e.g., a country, a planet, a solar system, or even a galaxy) where, all of a sudden, there appears an internal threat. This threat produces undesirable results that will be called here outcomes.

Let us reinforce the idea that the threat is internal to the domain. Therefore, the domain (with threat included) should be regarded as a closed system. The threat imposes to the domain a certain load. The creatures living inside the domain have obligatorily to accept this load and respond to it with outcomes. Since the threat is considered something bad, it is desirable that, somehow, the outcomes are kept at minimum levels.

To fight the threat, the creatures of the domain have some means of support that will be called here resources. The resources may be employed to combat the threat in different forms that will be called here factors. The factors compete among themselves for the resources in a non-collaborative way. Therefore, each resource may be used to deal with one factor or another, but not used simultaneously by more than one factor. 
The outcomes are countable numbers. The factors are countable and discrete numbers. They are different forms of allocating the resources to mitigate the threat. On the other hand, the resources may be either countable or uncountable. They may be either limited or unlimited. In the case of being limited, they pose restrictions that the solution to the problem must observe, i.e., it becomes a constrained optimization problem.

In summary, in this scenario an unexpected threat imposes an unavoidable burden on a certain domain. It is by all means desirable to minimize the amplitude of the outcomes. To reach this goal, the domain creatures use the available resources at their own will, but they wish to have some sort of guidelines to make sure that the resources are allocated for best cost/benefit ratio. With resources restrictions, the scenario acquires a complex nature. A rational solution is sought for this threat-outcome problem.

\section{The inspiration}

The hypothetical scenario described in the previous section may be likened in the continuum mechanics arena to the problem of a flexible structure undergoing dynamic loads. The structure is the open system (the plant to be analyzed or controlled), the loads are the threats (the actions being exerted upon the plant), and their sum is the total closed system (the domain).

Within these assumptions, one may invoke Hamilton's principle [1,2] as Lagrangian analysis tool, which may be stated as follows:

$\delta \int_{t_{1}}^{t_{2}} \mathcal{L} \mathrm{d} t+\int_{t_{1}}^{t_{2}} \delta \mathcal{W} \mathrm{d} t=0$

Here, $\mathcal{L}$ is the system's Lagrangian function, the difference between the system's total kinetic and potential energies, and $\delta \mathcal{W}$ is the virtual work done by external and nonconservative forces acting on the system.

In Eq. (1), $\mathcal{L}$ represents the system's internal dynamics, whereas $\mathcal{W}$ includes the effects of external excitations. Furthermore, $t_{1}$ and $t_{2}$ stand for two arbitrary time instants in which the state of the system is known. Finally, the symbol $\delta$ represents the mathematical operation of variation, which must be performed in the space of functions.
Ideas related to the calculus of variations have been developed for more than two centuries. During this period of time, these ideas have found application to practically all areas of the Exact Sciences, with focus on Physics, and Engineering [3]. For historical appraisals of this theme, the reader may consult Ferguson [4] and Goldstine [5]. For modern views on foundations and applications, the reader is referred to Berdichevsky $[6,7]$.

The main objective in this paper is to develop a theory based on variational calculus ideas to investigate complex scenarios requiring strategic decisionmaking, including applications in Social Sciences. In special, the purpose here is to apply the approach to study the current COVID-19 pandemic.

With this objective in mind, let us rewrite Eq. (1) as follows:

$\int_{t_{1}}^{t_{2}}(\delta \mathcal{L}+\delta \mathcal{W}) \mathrm{d} t=0$

Here, we have used the commutative property of the mathematical operations of integration and variation.

In the integrands of Eq. (2), $\mathcal{L}$ includes the system internal energies (kinetic and potential) developed from external excitations and $\delta \mathcal{W}$ represents the virtual work done by the external forces acting on the system. Energy and work have the same dimensional representation and they are interchangeable. In our model, the internal energies $\mathcal{L}$ are linked to system characteristics like stiffness or impedance, whereas $\mathcal{W}$ describes the action of the external threat.

In our planet, and perhaps in the whole universe, it is known that energy is expressed as a quadratic expression of the system variables. Once subjected to loads, system internal stresses are negotiated so that its equilibrium is achieved when the energy absorbed by the system reaches a minimum. This is the inspiration we use here to seek a solution for the proposed threatoutcome problem. It is simply Nature acting at its own manner.

Let us consider as domain a closed system. Internally to this system, there is a load resulting from a threat. The load acts within the system doing some work. The system absorbs the load, distributes the resulting stresses throughout itself, and provides an answer, generically identified as an outcome. It is wanted to establish some sort of correlation between the threat and the outcome and, if possible, to determine the outcome. 
Let us consider all forms of energy and work involved in this case and hypothesize that the sum of their contributions is written as a single functional $\mathcal{I}$. From Eq. (2), then, we get that the first variation of $\mathcal{I}$ is null, i.e.

$\delta \mathcal{I}=0$

By constructing a similarity with energy, let us assume in the general case the possibility of obtaining $\mathcal{I}$ as a quadratic function of all system variables. In this case, $\mathcal{I}$ is a positive definite functional. Within these hypotheses, the problem can be solved by minimizing $\mathcal{I}$ in the sense given by Eq. (3). Nature is such that this mathematical process leads to a minimum because it is known that the second variation of $\mathcal{I}$ is positive, i.e.

$\delta^{2} \mathcal{I}>0$

This is the concept used here to develop a solution to the threat-outcome problem. We imply that it is possible to write a functional in integral form for the problem and that it can be minimized in terms of the domain factors and system variables. To show how this works, let us discuss the concept in its mathematical details.

\section{The mathematics of the concept}

The threat-outcome problem is discussed here in general terms for a single functional $\mathcal{I}$. The case for multiple objective functions is presented as an extension in Sect. 6 of this paper.

Let us consider that the functional $\mathcal{I}$ can be expressed in three chained levels of relational dependence, as follows:

1. Competing factors-Let us consider, in first level, that $m$ groups of interest (stakeholders) dispute the governance of a given scenario. Let us indicate each of these groups by $\alpha_{i}, i=1,2, \ldots, m$. To each of these indicators, let us associate a value called factor criticality, within the domain $0 \leq \alpha_{i} \leq 1$. Finally, let us impose the following normalization condition:

$$
\sum_{i=1}^{m} \alpha_{i}=1
$$

2. System variables - Let us consider, in second level, that each competing factor $\alpha_{i}$ may be described in terms of $n$ system variables $x_{j}$, so that

$$
\alpha_{i}=\alpha_{i}\left(x_{j}\right) \quad i=1,2, \ldots, m \quad j=1,2, \ldots, n
$$

3. Time dependence-Let us consider, finally, in third level that each system variable $x_{j}$ may be time dependent, so that

$$
x_{j}=x_{j}(t) \quad j=1,2, \ldots, n
$$

With these preliminaries, let $\mathcal{I}$ represent a functional in integral form that can be expressed as a general quadratic form of factors $\alpha_{i} \quad(i=1,2, \ldots, m)$. Let us suppose that $\mathcal{I}$ obeys a stress-relieving principle like the Principle of Energy in the form of Eq. (3). The factors may be expressed in terms of variables $x_{j}(j=1,2, \ldots, n)$ which can also be functions of time $t$. With this enchained notation, Eq. (3) may be rewritten as follows:

$\delta \mathcal{I}\left(\alpha_{i}\left(x_{j}(t)\right)\right)=0 \quad i=1,2, \ldots, m \quad j=1,2, \ldots, n$

In the first level of analysis, we remain within the framework of factors $\alpha_{i}(i=1,2, \ldots, m)$ only, not considering the modeling details introduced by the system variables $x_{j}(j=1,2, \ldots, n)$. In this case, Eq. (8) can be presented as follows, using Einstein's sum notation:

$\frac{\partial \mathcal{I}}{\partial \alpha_{i}} \delta \alpha_{i}=0$

Since variations $\delta \alpha_{i}(i=1,2, \ldots, m)$ cannot be all simultaneously equal to zero, we end up with the following set of results:

$\frac{\partial \mathcal{I}}{\partial \alpha_{i}}=0 \quad i=1,2, \ldots, m$

If $\mathcal{I}$ is indeed a quadratic form of the factors $\alpha_{i}$, Eq. (10) represents a system of linear equations of order $m$. If $\mathcal{I}$ is of order higher than 2 , but still positive definite, Eq. (10) represents a set of $m$ nonlinear equations that can be solved by numerical methods. 
In the second level of analysis, we have first to expand all $m$ factors $\alpha_{i}$ in terms of the $n$ system variables $x_{j}$. Then, the problem of variation of $\mathcal{I}$ (Eq. 8) goes like this:

$\frac{\partial \mathcal{I}}{\partial \alpha_{i}} \frac{\partial \alpha_{i}}{\partial x_{j}} \delta x_{j}=0$

Since $\delta x_{j}(j=1,2, \ldots, n)$ cannot be all equal to zero, we obtain

$\frac{\partial \mathcal{I}}{\partial \alpha_{i}} \frac{\partial \alpha_{i}}{\partial x_{j}}=0 \quad j=1,2, \ldots, n$

which represents a system of linear or nonlinear coupled equations of order $n$ that can be solved by numerical methods.

In the third level of analysis, we consider that all variables $x_{j}$ may be functions of time $t$. If all $x_{j}$ are constant with time, we have a static scenario. In the general case, the scenario has dynamics described by

$x_{j}=x_{j}(t) \quad j=1,2, \ldots, n$

It is interesting to observe that in the first level of analysis, restricted to factors, time $t$ does not appear explicitly and the analysis seems to involve, at first sight, a static scenario. Within this level, the model might be enough for decision-making, especially if this is a strategic level. In the second level of analysis, time $t$ is implicitly included in the system variables $x_{j}(t)$ and the scenario full dynamics is revealed.

Equation (12) represents a set of $n$ coupled linear or nonlinear equations that can be used to describe the scenario dynamics. For instance, in the case with two factors and three system variables, the set expands as follows:

$$
\begin{aligned}
& \frac{\partial \mathcal{I}}{\partial \alpha_{1}} \frac{\partial \alpha_{1}}{\partial x_{1}}+\frac{\partial \mathcal{I}}{\partial \alpha_{2}} \frac{\partial \alpha_{2}}{\partial x_{1}}=0 \\
& \frac{\partial \mathcal{I}}{\partial \alpha_{1}} \frac{\partial \alpha_{1}}{\partial x_{2}}+\frac{\partial \mathcal{I}}{\partial \alpha_{2}} \frac{\partial \alpha_{2}}{\partial x_{2}}=0 \\
& \frac{\partial \mathcal{I}}{\partial \alpha_{1}} \frac{\partial \alpha_{1}}{\partial x_{3}}+\frac{\partial \mathcal{I}}{\partial \alpha_{2}} \frac{\partial \alpha_{2}}{\partial x_{3}}=0
\end{aligned}
$$

Once a solution is obtained for each of the $n$ variables $x_{j}(t)$, the time history of each of the $m$ factors $\alpha_{i}(t)$ can be constructed by reversing the sense of analysis. From these results, it is possible to simulate all the scenario dynamics, provided there is a complete set of initial and boundary conditions to yield a well-posed mathematical problem.

Extensions to the mathematical perspective of the concept presented here are given in Sect. 6 of this paper. Among the extensions, we consider the inclusion of restrictions applied to the system variables $x_{j}(t)$. Now, let us discuss the concept from another perspective.

\section{The physics of the concept}

Let us suppose, for the sake of simplicity that we have a scenario described by a single positive definite energylike functional $\mathcal{I}$, where the number of competing factors $\alpha_{i}$ is two, and that the amount of resources to face the threat is unlimited. Under these hypotheses, the threat-outcome problem can be developed on a plane for easy visualization.

Figure 1 depicts what we call here the baseline solution. The threat (or loading) is absorbed by the entire system in uniform fashion because there is no competition between the two factors. In mathematical terms, the two factors are independent of each other or, in practical terms, Factor 1 does not feel the existence of Factor 2 and vice-versa. This is the limiting case in which

$\delta^{2} \mathcal{I}=0$

The value of the outcome depends on the amplitude of the threat and on the system capability of absorbing this amplitude, something that in Engineering is called the system impedance or stiffness, depending on the system being electrical or mechanical. Let us denote this baseline outcome by $O_{b}$.

In order to obtain graphics like those displayed in Figs. 1, 2, 3 and 4 in this section, we need to map the functional $\mathcal{I}$ in terms of the generic argument $\sigma$ within the linear domain $-1 \leq \sigma \leq+1$. For this purpose, we make the following linear transformations:

$\alpha_{1}=\frac{1}{2}(1-\sigma) \quad$ and $\quad \alpha_{2}=\frac{1}{2}(1+\sigma)$

Note that these transformations observe the normalization condition (5), in the sense that the sum of the two Factors for any given value of $\sigma$ is equal to 1 . With these transformations, Factor $\alpha_{1}$ has the normalized value $1(100 \%)$ at $\sigma=-1$ and decreases to the value 


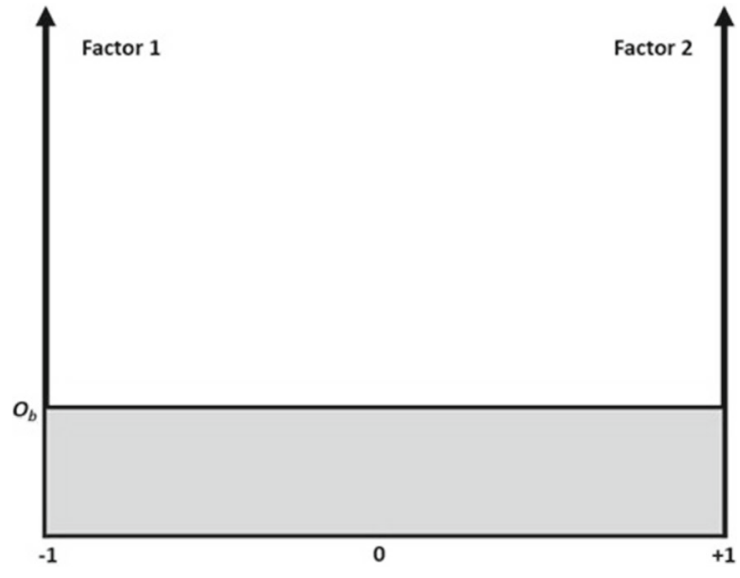

Fig. 1 Baseline unrestricted problem

$0(0 \%)$ at $\sigma=+1$, whereas Factor $\alpha_{2}$ has the value 0 at $\sigma=-1$ and increases to the value 1 at $\sigma=+1$. At the midpoint $\sigma=0$, both Factors have the same value $0.5(50 \%)$.

Figure 1 presents the competition between the two Factors. Value $\sigma=-1$ represents a condition where there is $0 \%$ weighting of Factor 2 and $100 \%$ weighting of Factor 1. Similarly, value $\sigma=+1$ represents the counterpart situation where there is $0 \%$ weighting of Factor 1 and 100\% weighting of Factor 2. Exactly in the middle, value $\sigma=0$ displays the condition where both Factors have the same weighting, that is, $50 \%$ for each one.

The baseline outcome $O_{b}$ represents the accepted minimum response of the system to the threat. This outcome is indifferent to the decision of facing the threat by giving more emphasis to one Factor or to the other because the resources are unlimited.

Now we pose a crucial question: what happens if we consider that one or more of the resources is in fact limited? In this case, the Factors start competing for the resources. In other words, one Factor becomes aware of the existence of the other. A well-educated guess comes to stage for the answer: the once straight line shown in Fig. 1 now becomes a curve with positive concavity, i.e., with positive second variation.

The reason for this behavior is not difficult to understand. If Factor 1 grabs more resources than Factor 2, the performance of both changes. Factor 1 will continue to display results not very different from those obtained in the unrestrained baseline case, whereas the performance of Factor 2 will be poorer. The sum of

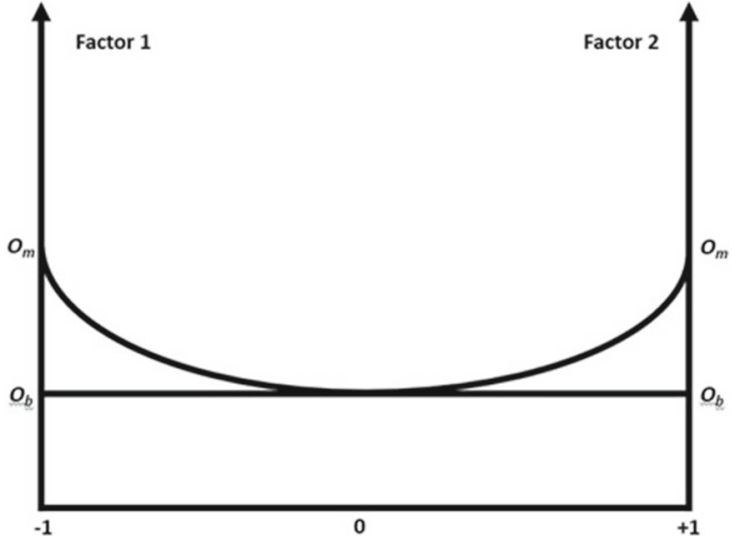

Fig. 2 Perfectly symmetrical competing problem

the performance of both Factors will be such as, for the same system impedance or stiffness, to increase the level of the undesirable outcomes. Therefore, the resulting curve goes above the $O_{b}$ level for any combination of limited resources distribution.

Figure 2 depicts the perfectly symmetrical case where both Factors have similar competences in gathering resources for themselves and similar performances in applying them to give the threat a combat. Besides, we consider here that they both yield the same maximum outcome $O_{m}$ when they obtain $100 \%$ of all system resources they can take.

In Fig. 2, the abscissa $\sigma=-1$ represents the condition where it is given 100\% emphasis to Factor 1 and $0 \%$ emphasis to Factor 2 . Thus, the difference between the outcomes $O_{m}$ and $O_{b}$ is mainly due to the decision of underemphasizing Factor 2. Similar considerations, like in a mirror, are implied for the condition given in the abscissa $\sigma=+1$.

In this case, there is no doubt that the minimum outcome happens when equal attention is given to both Factors and that, in this condition, $\alpha_{1}$ values the same as $\alpha_{2}$. For the sake of simplicity, we consider here that the $50-50 \%$ split condition yields as minimum outcome the baseline value $O_{b}$, but this is not an obligatory result. In fact, it is an optimistic result. Most probably, the existence of competition for resources between the two Factors will produce a minimum outcome value that is higher than $O_{b}$.

Figure 3 shows an asymmetrical case of competition between the two Factors. In this figure, the maximum outcome $O_{m_{1}}$ of Factor 1 is bigger than the maximum outcome $\mathrm{O}_{m_{2}}$ of Factor 2. Besides, the minimum of 


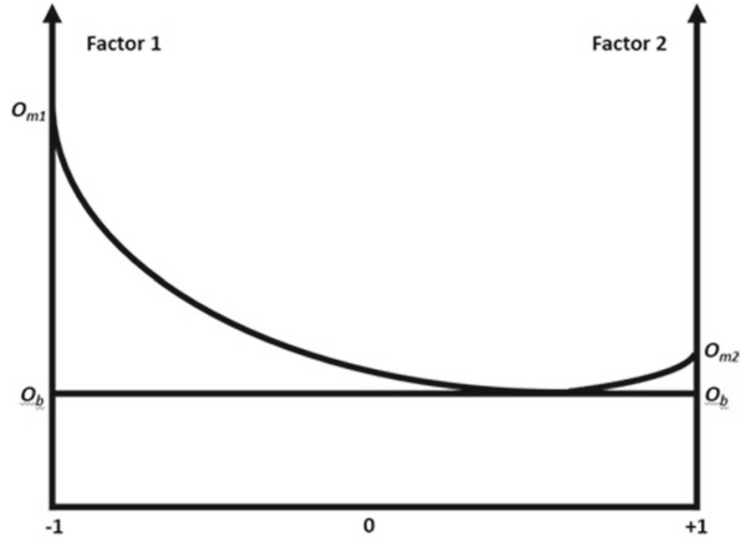

Fig. 3 An asymmetrical competing problem

the curve happens between the midpoint $\sigma=0$ and the $\sigma=+1$ point. This means that the impact of Factor 2 on the maximum outcome $O_{m_{1}}$ is bigger than the impact of Factor 1 on the maximum outcome $\mathrm{O}_{m_{2}}$. In this case, Factor 2 is identified as being more critical than Factor 1, i.e., that $\alpha_{2}>\alpha_{1}$.

The idea in the asymmetric case is altruistic, in the sense that the more resources you bring to your factor, the more deficient results you collect that come from the decision of neglecting the other factor. In Fig. 3, Factor 1 is less critical than Factor 2 because placing emphasis on Factor 1 yields greater outcomes than placing emphasis on Factor 2. Therefore, in order to minimize the outcomes, it makes more sense to invest in Factor 2 than in Factor 1.

Figure 4 shows the limiting asymmetrical case where the minimum occurs at the $\sigma=+1$ point. The totality of the criticality of the system is placed on Factor 2. In this case, if all resources are invested on Factor 2, the outcome is, for the sake of simplicity, the same of the baseline case $\left(O_{b}\right)$. On the other hand, if all resources are invested on Factor 1, the maximum outcome $O_{m}$ is bigger than the baseline value $O_{b}$. In other words, the final outcome is inherited from the criticality of Factor 2, from the fact of Factor 2 having been deprived from needed critical resources by the decision maker.

The final goal in this threat-outcome problem is to obtain the minimum outcome. For such condition, it is absolutely necessary to discover which Factor is the critical one and to invest more on it. Since the case depicted in Fig. 4 is an ideal limit, it is worth discover-

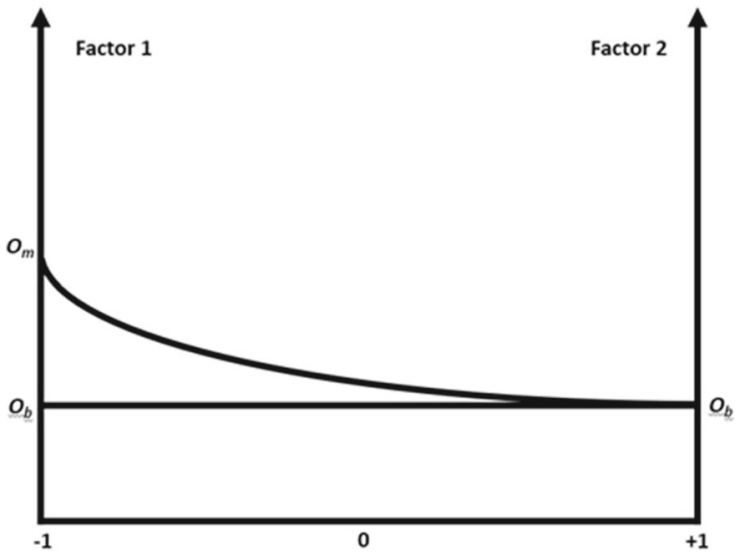

Fig. 4 A limiting asymmetrical competing problem

ing in a real problem where the minimum point is, like in the cases shown in Figs. 2 or 3.

Having discussed the mathematics and the physics of the concept, let us now present its application to the WHO SARS-COV-2 (COVID-19) case.

\section{Application to the COVID-19 case}

Let us consider the hypothetical situation in which a country is invaded by a virus outbreak, subsequently elevated to pandemic status. This is an undesirable threat. People fear its outcomes because vaccines are limited and there is no way out. The threat must be faced with all the country's resources. Fortunately, the country possesses a set of means for mitigating the threat. People want to use them well, but they wonder which is the best way of doing it.

\subsection{The scenario without competition}

Let us start the analysis in search for an answer to this threat-outcome problem. Let us consider the baseline solution, the one in which the country has no vaccine, but has all the remainder needed resources, and better yet, they are unlimited. In this ideal scenario, there is little modification in the country's modus vivendi, for the country's resources are plentiful. To name a few:

1. Infrastructure-hospitals, health centers, ambulatories, clinics, all well installed, geographically well distributed, and with sufficient beds either in infirmary or ICU; 
2. Logistics-sufficient means of transportation for people and delivery of supplies in order to make all subsystems to function flawlessly;

3. Personnel — doctors, nurses, and attendants, all welleducated, prepared, and trained, in enough quantity to offer prompt services with quality;

4. Equipment-adequate equipment and working supplies provided by national industries as needed, in the short term, at fair prices;

5. Treatment - effective drugs and therapies with approved protocols for the clinical care of the patients, despite the novelty of the disease;

6. Budget - sufficient money to pay for all services and emergency aids at all levels of the Executive Branch, federal, state, and county;

7. Policies-public authorities of all government branches working in harmony, each of them in their specific roles, supporting each other;

8. Living conditions - enough food, plenty of energy, adequate sanitation, and everything else to overcome the transitions imposed by the threat;

9. Guidance to the population-orientation on how people should behave to reduce contamination, decrease chances of getting sick, seek proper attendance, etc.; and

10. People's will-people and institution's intentions to fight the threat, to minimize the number of deaths, and to prepare society for the future.

This is a non-exhaustive list of resource groups that may be used to generate mathematical model parts and to identify variables for the threat-outcome problem. They provide a taxonomy to keep in reference: infrastructure, logistics, personnel, equipment, treatment, money, political support, living conditions, sanitary orientation, and people's will.

In this ideal scenario, until approved vaccines come out and become effective, loss of lives due to the pandemic have to be accepted-and mourned-by society as an unfortunate but natural phenomenon. Besides the health-related orientations, there is no need for any other measures and everyone's lives go on as normal as possible. Furthermore, there is little to question from the economic and from the psychosocial standpoints.

The previous list of resources can be cast, for the sake of systematization, in two Factors: Health $\left(\alpha_{1}\right)$ and Economy $\left(\alpha_{2}\right)$. It can be realized that some resources have very strong relation to Factor 1; others, to Factor 2 . However, one can also realize that some of them have relational links to both Factors, which means that the problem is definitively coupled.

In the hypothetical scenario, if we create a graph of the total number of deaths due to the pandemic on the vertical axis versus prioritizing the Health and Economy Factors in the horizontal axis, the result is a horizontal line going from the $100 \%$ Health / $0 \%$ Economy to the $0 \%$ Health / $100 \%$ Economy point. In other words, the graph is exactly similar to the horizontal line presented in Fig. 1, extending from the $\sigma=-1$ till the $\sigma=+1$ point.

In the ideal scenario, with plentiful of resources, society, seen as a system, is not very much stressed and the number of deaths $O_{b}$ does not make, unfortunately, newspapers headlines with very big letters. In other words, there is no competition between the two Factors and the death toll is accepted by society as a situation imposed by Nature.

In order to lower the number of deaths $O_{b}$, the general recommendation is to make investments in Education, Science, Technology, and Innovation (EST\&I). These investments have structuring capabilities, in the sense that they can improve the performance of all groups of resources. This is equivalent to increasing society robustness (impedance or stiffness) as a whole and, thus, to lowering the system outcomes.

\subsection{The scenario with competition}

The perfect scenario described in the previous subsection begins to change when one realizes that resources are finite and that some sort of restrictions may be applied, either on Health $\left(\alpha_{1}\right)$, on Economy $\left(\alpha_{2}\right)$, or on both. Modeling on the Health Factor seems to be simpler, based on more tangible indicators, variables and restrictions. Modeling on the Economy Factor seems to be more difficult, as it is based on many intangible indicators.

In the real world, infrastructure is lacking, logistics has flaws, there is not enough personnel, equipment and supplies become expensive, there are no medicines or acknowledged protocols for treatment of a new disease, and budget misses timing and magnitude. In addition, political actions are confusing and non-collaborative $[8,9]$ living conditions become poorer, people have no access to proper and opportune guidance, and people's will fades, leading in some cases to depression or even to suicide. 
All these elements can be individually modeled in quadratic forms and these forms can be weighted and integrated to compose factors that are components of the integral functional $\mathcal{I}$ to be minimized.

For both Factors, there are several elements (resources, variables, and restrictions) to consider in the model. Let us take, for instance, the number of ICU beds as an element. In countries where there are plenty of them, they may be regarded as resources and, perhaps, as variables. In countries where they are lacking, they should be regarded as restrictions. The same perception stands for lung respirators and respiration tents.

By placing strong emphasis on the restrictions, some authorities have used the possibility of collapse of their country's Health Systems to justify the adoption of rigid social isolation measures. However, these measures harm the country's economics.

Let us consider as general economic losses the closure of companies, the rising of unemployment, the starvation, and resulting deaths. Let us add the depression caused by isolation, the conflicts resulting in fights and deaths. Let us also consider the psychological effects on people due to isolation, some leading to suicide. And the list continues. Therefore, as much as there are deaths due to the virus because of health conditions, there are deaths due to the virus motivated by economic reasons.

Without having developed very detailed models of the whole problem, including all possible elements from both Factors, authorities have only partial, fuzzy, or even meaningless knowledge to base their decisions upon. The point is that, as of now, it is unclear which of the two Factors is the critical one in the sense discussed in this paper. Until we have enough analyses, results, and field evidences to clarify this issue, the wisest position to take is to consider that we have in hand a symmetric case.

Anyway, it makes reasonable sense to consider that in the real case there is an optimum point to minimize the number of deaths, like those points appearing in Figs. 2 or 3. For the sake of equilibrium and justice, considering both Factors equally critical, here we will take the symmetric case (Fig. 2) to obtain a qualitative closure of this study and to reach some conclusions.

\subsection{A mathematical application}

The idea developed in this article is in its infancy. However, there is condition now to make an application at the first (factor) level of analysis in order to demonstrate how the concept works.

Let us consider as the threat-outcome problem functional a general and complete quadratic form of Factors $\alpha_{1}$ (Health) and $\alpha_{2}$ (Economy). The complete equation of a conic reads as follows:

$\mathcal{I}\left(\alpha_{1}, \alpha_{2}\right)=A \alpha_{1}^{2}+B \alpha_{2}^{2}+C \alpha_{1} \alpha_{2}+D \alpha_{1}+E \alpha_{2}+F$

Here, the coupling between the two Factors appears explicitly in the term leaded by the coefficient $C$. There are, in the general case, six coefficients to be determined.

The application of Eq. (9) to this scenario yields the following result by collecting the terms with $\delta \alpha_{1}$ and $\delta \alpha_{2}$ in the expansion:

$$
\begin{aligned}
\delta \mathcal{I}= & \left(2 A \alpha_{1}+C \alpha_{2}+D\right) \delta \alpha_{1} \\
& +\left(2 B \alpha_{2}+C \alpha_{1}+E\right) \delta \alpha_{2}=0
\end{aligned}
$$

Since the variations $\delta \alpha_{1}$ and $\delta \alpha_{2}$ are both different from zero in the general case, the two parentheses in Eq. (20) must be set each one equal to zero. In consequence, we obtain the following set of equations to determine the optimized values of the Factors' criticalities:

$$
\left[\begin{array}{cc}
2 A & C \\
C & 2
\end{array}\right]\left\{\begin{array}{l}
\alpha_{1} \\
\alpha_{2}
\end{array}\right\}_{o}=\left\{\begin{array}{l}
-D \\
-E
\end{array}\right\}
$$

from where we get

$$
\alpha_{1 o}=\frac{2 B D-C E}{C^{2}-4 A B} \quad \text { and } \quad \alpha_{2 o}=\frac{2 A E-C D}{C^{2}-4 A B}
$$

These are the values for $\alpha_{1}$ and $\alpha_{2}$ where the minimum of the functional $\mathcal{I}$ occurs. By proper modeling of the Factors according to the resources described previously, numbers can be assigned to the coefficients $A$ through $F$. From these assignments, we can determine the optimized values of $\alpha_{1 o}$ and $\alpha_{2 o}$ and identify which of the two Factors is the most critical one. The better the modeling represents reality, the greater the quality 
of the optimized results to substantiate decisions to be taken over the scenario.

The application to the symmetric case of dynamic equilibrium between the two Factors (Health and Economy) goes as follows. The two parentheses inside Eq. (20) must be set equal to zero, observing that $\alpha_{1}$ and $\alpha_{2}$ must be both made equal to 0.5 , which is equivalent to $50 \%$ of emphasis for each Factor. From this operation we obtain these two relations:

$2 A+C+2 D=0 \quad$ and $\quad 2 B+C+2 E=0$

If we substitute the linear transformations (18) for $\alpha_{1}$ and $\alpha_{2}$ in the functional $\mathcal{I}$ of Eq. (19), this quadratic form is transformed into a new quadratic form $\mathcal{I}_{m}$, with subscript $m$ for mapping, given in terms of $\sigma$ by

$$
\begin{aligned}
\mathcal{I}_{m}(\sigma)= & \frac{1}{4}\left[A(1-\sigma)^{2}+B(1+\sigma)^{2}+C\left(1-\sigma^{2}\right)\right. \\
& +2 D(1-\sigma)+2 E(1+\sigma)+4 F]
\end{aligned}
$$

There is no loss of generality if we normalize the quadratic form by setting $A=1$ and $F=0$. For the determination of the remainder coefficients $B, C, D$, and $E$, in the symmetric case discussed in the previous section, we invoke the two optimization conditions (23) and two non-redundant boundary conditions which yield equal maximum outcome $O_{m}$ at the extremities $\sigma=-1$ or $\sigma=+1$, and minimum outcome $O_{b}$ at the midpoint $\sigma=0$.

After clearing the algebra, we obtain the following results for the coefficients of Eq. (24):

$$
\begin{aligned}
& A=1 \\
& B=2 O_{m}-4 O_{b}-1 \\
& C=-2 O_{m} \\
& D=O_{m}-1 \\
& E=4 O_{b}-O_{m}+1 \\
& F=0
\end{aligned}
$$

These results yield a curve similar to the one presented in Fig. 2. Besides, they confirm that we obtain a minimum at the midpoint $\sigma=0$, provided $O_{m}>O_{b}$, i.e.,

$$
\frac{\mathrm{d}^{2} \mathcal{I}_{m}}{\mathrm{~d} \sigma^{2}}(0)=\frac{1}{2}(A+B-C)=2\left(O_{m}-O_{b}\right)>0
$$

In the limit where $O_{m}=O_{b}$, we obtain

$$
\frac{\mathrm{d}^{2} \mathcal{I}_{m}}{\mathrm{~d} \sigma^{2}}(0)=\frac{1}{2}(A+B-C)=2\left(O_{m}-O_{b}\right)=0
$$

which is the unrestricted baseline case discussed in the previous section, presented in Eq. (17), and displayed in Fig. 1.

\subsection{Relation to SIR-like epidemic models}

The threat-outcome problem is introduced in this paper in general terms. The application to an epidemic or pandemic is a case of immediate concern that has prompted this study, but this theory is not limited to this scenario.

For a recent appraisal of the mathematical tools available for the study of epidemics, the reader is referred to Yates [10]. The most used mathematical approach to treat this problem has been inaugurated in 1927 by Kermack and McKendrick [11]. It divides the domain of people threatened by an epidemic in three groups: the Susceptibles (S), the Infectives (I), and the Removed (R), including people recovered and deceased. It is referred to as the SIR model.

There are now at least a dozen variants of the SIR traditional model. This issue has been explored so intensively and rapidly in the last year due to the COVID-19 pandemic that good references for a fast track on the subject can be found in the open library Wikipedia, e.g., [12] and [13].

Let us consider the SIRD variant applied to a domain in which there is a group of $N$ individuals subjected to an epidemic. This group is divided in four classes of people, the Susceptibles $S$, the Infectives $I$, the Recovered $R$, and the Deceased $D$. The dynamic constraint of the group is that, at any given time instant $t$, the following relation is valid:

$N(t)=S(t)+I(t)+R(t)+D(t)$

This model includes the so-called vital dynamics, in the sense that $N(t)$ may incorporate births and deaths since the beginning of the process. The initial values of individuals in each class are the following:

$S(0)=S_{0} \quad$ and $\quad I(0)=I_{0}$ 
with

$N(0)=S_{0}+I_{0}$

and

$R(0)=D(0)=0$

The SIRD epidemiological model is governed by the following set of nonlinear differential equations:

$$
\begin{aligned}
& \frac{\mathrm{d} S}{\mathrm{~d} t}=-\beta \frac{S I}{N} \\
& \frac{\mathrm{d} I}{\mathrm{~d} t}=\beta \frac{S I}{N}-\left(\gamma_{1}+\gamma_{2}\right) I \\
& \frac{\mathrm{d} R}{\mathrm{~d} t}=\gamma_{1} I \\
& \frac{\mathrm{d} D}{\mathrm{~d} t}=\gamma_{2} I
\end{aligned}
$$

In this model, $\beta$ represents the rate at which people become infected, the transmission rate of the disease, describing the migration of people from the $S$ to the $I$ class. Likewise, parameters $\gamma_{1}$ and $\gamma_{2}$ describe the rates at which infected individuals change class to recovered $R$ or deceased $D$, respectively. These parameters have to be inferred directly from experience or adjusted from measured data or available statistics.

What is expected, typically, in most cases, is that in this nonlinear problem class $S$ decreases, while class $R$ increases monotonically as time advances. Furthermore, class $I$ grows up, reaches a peak. and recedes to a lower level. In the original SIR model there was no provision for deaths, because the epidemic was considered mild. Subsequently, the model included class $D$ as an important element of vital dynamics.

The SIRD model has been used recently to evaluate the progress of the COVID-19 pandemic in Italy (Calafiore et al. [14]). The authors have incorporated two non-standard features to the development, namely, model parameters which are time-varying and identified directly from the available data. Another recent study has been devoted to the progress of the COVID19 outbreak in India (Chatterjee at al. [15]). The authors have observed the high sensitivity of the results to the variation in the model parameters values.

For almost a century now, the SIR model and its variants have been used as the main analysis tool of this theme. As shown in Eq. 33 through 40, it is represented by a coupled set of nonlinear ordinary differential equations with initial conditions, which can be integrated numerically to obtain the classes evolutions in time. This represents the state-of-the-art.

The concept presented in this paper is novel, in the sense that, regarding Mathematics, it is an integral, rather than a differential method. It can not only reproduce the dynamics of the SIR model but also incorporate the allocation of resources as an advantage. It can provide elements of analysis for strategic decision-making, something the SIR-like models cannot. Finally, it can be applied to any threat-outcome scenario, being not limited to an epidemic. Therefore, SIR-like models are limited in scope and applications, whereas the threat-outcome model is general and comprehensive in scope.

In summary, whereas the SIRD model is a mathematical tool for evolution tracking of four different classes of people in an epidemic, the threatoutcome approach is an integral optimization method for resources allocation and strategic decision. Both can handle the dynamics of people's classes during a pandemic. However, this is the only feature the SIRD model can offer to the analyst. The threat-outcome approach has much more to offer in terms of analytical capabilities.

This discussion on the SIRD model has been included here to provide support to the social isolation analysis to be presented in the sequence.

\subsection{Discussion on social isolation and non-pharmaceutical interventions}

Even with vaccines available for the COVID-19 virus, the definitive solution still calls for herd immunity. Presently and understandably, in several countries more emphasis has been given to the Health Factor than to the Economy Factor. Inside this implicit emphasis is the explicit model of social isolation. Some authorities affirm that, in order to save lives, social isolation should be horizontal, if not total lockdown. Some countries, on the other hand, have applied vertical isolation or even no isolation at all.

The comparison between the two positions brings no conclusion because it has been based on incomplete premises or because it is not possible to conduct valid experiments with proper and accepted controls. One has first to model the lives lost due to economic effects and compare to the lives lost due to the virus and to other untreated diseases. So far, to our knowledge, no studies 
of this kind have been performed with comprehensive and balanced approaches.

To apply horizontal social isolation or even lockdown is equivalent to place $100 \%$ emphasis on the Health Factor. We have shown previously in this paper that this is not the optimum solution, for it raises the total number of deaths. The optimum 50\%-50\% (split) symmetric solution suggests an equivalence to the vertical isolation model. Therefore, in order to save lives, the recommendation is to increase the emphasis on the Economy Factor. This decision implies a reduction in the total system stresses, bringing the system to a dynamic equilibrium, and building a win-win condition. Equilibrium here means that the grand total stress of the system will be lower than it has been since the beginning of the pandemic in most of the countries.

The best solution, therefore, is not in a model of horizontal and radical social isolation nor in a model of total openness. Isolation should be applied to people who are in health conditions called at risk, with comorbidities. Although without definitive quantitative results at the moment, but with reasonable scientific evidence, it seems that the optimum point is close to an intermediate social opening, controlled by the isolation of groups of people of higher risk and opening of groups of people of lower risk.

As early as of May 2020, it was already known that full lockdown strategies were ineffective in slowing down the COVID-19 outbreak (Meunier [16]). The author has found no change in the growth rate, doubling time, and reproduction number before and after the lockdown in countries like Italy, France, Spain, and United Kingdom. Extrapolations of the death toll in the absence of any lockdown policies have shown that these strategies might not have saved any life in western Europe. Furthermore, the study has shown that neighboring countries applying less restrictive measures have experienced a very similar epidemic evolution. If lockdowns do not help the Health Factor, why use them to harm the Economy Factor?

To confirm this evidence, similar conclusions can be drawn from the recent work of Acemoglu et al. [17]. The authors have studied via SIR models the effects of social isolation on the number of deaths and on the United States GDP reduction. They have divided the population of Infectives (I) between groups by age: young, middle aged, and old, with different reactions when infected. They have concluded that, for constant GDP reduction, there is significative decrease in the number of deaths by using vertical isolation in comparison to any other type of isolation or lockdown.

Another independent confirmation of similar understanding has been recently published by Bendavid et al. [18]. The authors have compared the spread of COVID-19 between countries where more restrictive Non-Pharmaceutical Interventions (mrNPIs) have been applied with countries where less-restrictive NonPharmaceutical Interventions (lrNPIs) have been in effect. In the first group they have included England, France, Germany, Iran, Italy, Netherlands, Spain, and the United States. For the second group they have selected South Korea and Sweden. The authors have not found significant benefits by enforcing mrNPIs. In other words, similar reductions in spread of the disease and on deaths have been achieved with lrNPIs.

Four studies about the inefficiency of lockdown implementations to control the spread of the COVID19 virus appear in an article by Miltimore [19]. These studies involve authoritative sources like the journals The Lancet and Frontiers in Public Health, and the Universities of Tel Aviv and Oxford. But the list does not end here.

Twenty-nine other independent studies, some using SIR-like models, present evidence and reinforce the conclusion that strict isolation measures like lockdowns, besides harming the Economy Factor, have little or no effect in controlling the spread of the COVID-19 pandemic. In other words, they are not useful either for helping the Health Factor. These studies, the majority still in preprint phase, have been compiled by the staff of the American Institute for Economic Research (AIER) in the article Lockdowns do not Control the Coronavirus: The Evidence [20].

A very strong confirmation of this finding has been recently given by Haug et al. [21] on the spread of the virus. The authors have quantified the effectiveness of 6068 hierarchically coded NPIs with data obtained from 79 territories and validated from 42,151 additional NPIs from 226 countries, i.e., a very sizable database. They have used computational techniques, statistical, inference, and artificial intelligence tools to treat the collected data. They have concluded that "Less disruptive and costly NPIs can be as effective as more intrusive, drastic ones," as a national lockdown.

Other recent discussions in the literature seem to support this position of bringing Health and Economy to a best, compromised equilibrium. Diffenbaugh et al. [22] discuss the long-term effects of the virus on 
Earth as a system. They say that "Restrictions to reduce human interaction have helped to avoid greater suffering and death from the COVID-19 pandemic, but have also created socioeconomic hardship." McKee and Stuckler [23] go beyond by making a statement that "If the world fails to protect the economy, COVID19 will damage health not just now but also in the future." Haldane and Chowla [24] complete the picture by choosing fast economic indicators to get an immediate picture of the COVID-19 impact on global Macroeconomics.

AIER has recently disclosed an appraisal of the pandemic scenario [25]. The Institute's staff report not only on Economy and Healthcare, but also on other important issues like mental health, hunger and poverty, unemployment, education, food and hospitality, and crime. The scenario is so encompassing in terms of issues that strategic decisions based just on a few aspects of the problem seem to be quite inadequate.

By the time new COVID-19 strains defy the positive impact of vaccines and make several countries on Earth to plunge into scenarios with increased cases of virus dissemination, authorities are tempted to decree lockdowns or severe social isolation measures as solutions to avoid their health systems collapses. The present study indicates that this is not the best track to follow to save lives in the short term and also in the long run.

In summary, we have provided here strong evidence that, to minimize deaths directly and indirectly related to the COVID-19 pandemic, the best strategy is to balance Health and Economy interventions in society. In practice, this implies that mrNPIs like lockdowns, stay-at-home orders, and business closures should be replaced by lrNPIs like social distancing guidelines, travel control, large gatherings bans, and isolation of infected cases. For other ideas, one may consult the database used in [21].

With this discussion closure, let us examine more extensions to the concept proposed in this paper.

\section{Possible extensions to the original concept}

The problem discussed in this paper may be extended to the case of multiple threats, multiple factors, with interconnected multiple restrictions, trying to reach multiple objectives with different weights. Another extension is the possibility of considering some sort of collaboration between the factors in the sharing of the resources, increasing the coupling between the problem elements. In addition, the problem can be even further generalized by making all these elements time-varying, transforming static into dynamic scenarios.

Here we have discussed at the conception level the two-dimensional case that can be visualized on a plane. With three dimensions, the solution can be seen in space. However, there is no limitation in going into the mathematical $N$-dimensional space. Either in the static or in the dynamic cases, the model will continue to represent a complex multidimensional minimization problem with or without constraints.

In this section, we present elements of discussion of two issues: inclusion of restrictions in the system's variables, and models with multi-objective functions and multiple factors.

\subsection{Inclusion of restrictions}

Let us consider a functional $\mathcal{I}$ subjected to a variational principle like the one given in Eq. (8). Let us suppose that both factors $\alpha_{i}(i=1,2, \ldots, m)$ and system variables $x_{j}(j=1,2, \ldots, n)$, included in the functional $\mathcal{I}$ may suffer dynamic restrictions $\rho_{k}(k=1,2, \ldots, p)$ in the form

$$
\begin{aligned}
& \rho_{k}=\rho_{k}\left(\alpha_{i}, x_{j}, t\right) \quad i=1,2, \ldots, m \\
& j=1,2, \ldots, n \quad k=1,2, \ldots, p
\end{aligned}
$$

The subject of constrained optimization is classical in the literature. For instance, consider as references the book by Bertsekas [26] and the recent tutorial by Klein [27]. The incorporation of restrictions into $\mathcal{I}$ can be made through $p$ Lagrange multipliers $\lambda_{k}$ that change Eq. (8) to

$$
\delta\left(\mathcal{I}-\lambda_{k} \rho_{k}\right)=0 \quad k=1,2, \ldots, p
$$

Here it is assumed that the products $\lambda_{k} \rho_{k}$ are expressed in the same dimensional form of the integral functional $\mathcal{I}$. This implies that if $\mathcal{I}$ is energy, so must be every weighted restriction $\lambda_{k} \rho_{k}$. This requirement does not pose great challenges, since the necessary dimensional adjustments can be inputted to every multiplier $\lambda_{k}$.

Again, for the sake of brevity, we will present here just the first level of analysis at the strategic (factor) level. The variation of Eq. (42) yields the following result: 


$$
\frac{\partial \mathcal{I}}{\partial \alpha_{i}}-\lambda_{k} \frac{\partial \rho_{k}}{\partial \alpha_{i}}=0 \quad i=1,2, \ldots, m \quad k=1,2, \ldots, p
$$

which represents a set of $m$ linear or nonlinear equations, depending on the mathematical representation of $\mathcal{I}$ and of each restriction $\rho_{k}$.

The interesting property of the Lagrange multiplier method is that it transforms a more complex constrained problem into a simpler unconstrained problem. The price to pay for this transformation is an increase in the number of unknowns.

\subsection{Multi-objective functions and multi-factors case}

The general functional $\mathcal{I}$ represents the sum of all the energies of the domain, system, and threat. It may be, in the general case, represented by the sum of two or more objective functions. For instance, the simultaneous minimization of number of deaths and of GDP reduction due to the pandemic appears in the study of Acemoglu et al. [17].

Here, for the purpose of discussion, let us consider the case where the problem has two objective functions $\mathcal{I}_{1}$ and $\mathcal{I}_{2}$ weighted by a single weight $w$, so that $0 \leq$ $w \leq 1$, and two competing factors $\alpha_{1}$ and $\alpha_{2}$, so that

$\mathcal{I}\left(\alpha_{1}, \alpha_{2}\right)=w \mathcal{I}_{1}\left(\alpha_{1}, \alpha_{2}\right)+(1-w) \mathcal{I}_{2}\left(\alpha_{1}, \alpha_{2}\right)$

The case of a single functional is recovered if the weight $w$ is set exactly equal either to 0 or to 1 . In this weighted case, the first level Eq. (9) is generalized to

$$
\left[w \frac{\partial \mathcal{I}_{1}}{\partial \alpha_{i}}+(1-w) \frac{\partial \mathcal{I}_{2}}{\partial \alpha_{i}}\right] \delta \alpha_{i}=0 \quad i=1,2
$$

which can be rewritten in extended form as

$$
\begin{aligned}
& {\left[w \frac{\partial \mathcal{I}_{1}}{\partial \alpha_{1}}+(1-w) \frac{\partial \mathcal{I}_{2}}{\partial \alpha_{1}}\right] \delta \alpha_{1}} \\
& \quad+\left[w \frac{\partial \mathcal{I}_{1}}{\partial \alpha_{2}}+(1-w) \frac{\partial \mathcal{I}_{2}}{\partial \alpha_{2}}\right] \delta \alpha_{2}=0
\end{aligned}
$$

Since the variations of $\alpha_{i}(i=1,2)$ cannot be both simultaneously equal to zero, the result is the following set of equations:

$$
\begin{aligned}
\frac{\partial \mathcal{I}}{\partial \alpha_{1}} & =w \frac{\partial \mathcal{I}_{1}}{\partial \alpha_{1}}+(1-w) \frac{\partial \mathcal{I}_{2}}{\partial \alpha_{1}}=0 \\
\frac{\partial \mathcal{I}}{\partial \alpha_{2}} & =w \frac{\partial \mathcal{I}_{1}}{\partial \alpha_{2}}+(1-w) \frac{\partial \mathcal{I}_{2}}{\partial \alpha_{2}}=0
\end{aligned}
$$

This is a coupled set of two linear or nonlinear equations, depending on the nature of the two objective functions $\mathcal{I}_{1}$ and $\mathcal{I}_{2}$.

The discussion in this paper has been mostly qualitative, for this is an introductory study in the conceptual level. Further extensions may consider the modeling of factors, system variables, and restrictions, in order to allow for computational simulations. Once these results become handy, they can be confronted with field data for model validations.

Another extension may consider the inclusion in the analysis of a third factor, e.g., a factor linked to social issues. This model will provide a more general and balanced representation of the real scenario humanity faces now with the COVID-19 threat. This and other themes may be explored in future studies about the integral optimization approach introduced in this paper.

\section{Concluding remarks}

Out of clutter, find simplicity. Albert Einstein

This is a contribution of inter and multidisciplinary nature. It does not belong to Engineering, Medical or Social Sciences alone. Its biggest merit is just that, to allow the study of a subject in the intersection of these Sciences considered independently. We have taken the initial inspiration from Engineering to handle a strategic conflict between the Health and Economy Factors. In the common ground, when the mathematical models are constructed, we have been dealing with the dynamics of nonlinear systems.

As usually happens in multidisciplinary approaches, it is not possible to use the most evolved model of each of the areas intertwined. Therefore, some simple tools of each area are invoked and integrated into a single scenario.

A theory to allow macroanalysis of complex systems facing threats with competing factors and restrictions has been presented. The systems are stressed by threats and their outcomes are undesirable. The central idea of this research has been to find the best rational way to employ resources within the competing factors to minimize the outcomes. 
The model has three chained levels of analysis: the strategic, the functional, and the evolutional. The strategic level is described by factors of large scale of integration. In this level the scenario appears to be simple and static. Factors are composed by functions, which build up the second scale of analysis. Time appears explicitly in the third scale, for the functions may be time-dependent. Since this article introduces the idea, most of the discussion here has been conducted in the first, strategic scale of the model.

To start, a baseline problem has been established by considering the ideal case in which the competing factors have no restrictions or limits. From the baseline benchmark, a theory has been constructed to deal with more general problems inspired on Hamilton's Principle of Stationary Action.

The mathematical model of the problem has been applied in the first level of analysis, which is sufficient for strategic decision-making. Despite the complexity of the scenario, it is shown here that the resulting model and the final solution are simple, as indicated by Einstein's quote.

The theory has been applied to the case of the COVID-19 virus as a threat to a country with two competing Factors: Health and Economy. Within the hypotheses and qualitative conditions established by the model, it is shown that, in order to minimize the total number of deaths produced by the virus, the best recommendation is to balance the emphasis on both Factors, by using their respective criticalities as weights. This conclusion implies, for instance, that a vertical social isolation model and other less restricted interventions should be enforced to bring the system stresses to equilibrium and, in consequence, the total number of deaths to a minimum.

The social isolation model is not the only possibility, for the Economy Factor has several other variables and restrictions to be considered and analyzed. This is an open question, like many others created within the conception introduced in this article.

The Mathematics used to analyze problems of epidemics has been based on the solution of differential equations of the SIR model [11]. In this paper, a different approach has been proposed, based on the optimization of an integral positive definite functional likened to energy. The approach used here is more general, in the sense that it can deal with the allocation of resources and provide useful and more comprehensive information for decision-making.
The model presented here can be extended to more general cases. It can be developed further to evolve from the present qualitative to a future quantitative status. Then, it may allow for computational simulation and comparison with field statistics for validation and forecasting. In this way, it may become a useful tool for authorities charged with strategic decision-making in complex scenarios by reducing uncertainty and guessing.

Acknowledgements The author acknowledges discussions while preparing early versions of this paper with Professors Mauro Taschner, Odylio Denys Aguiar, Luciano Humberto Lampi, Leon Chant Dakessian, Denisard Cneio de Oliveira Alves, and Dewey Harper Hodges.

\section{Declarations}

Conflict of interest The author declares that he has no conflict of interest.

\section{References}

1. Goldstein, H.: Classical Mechanics, 2nd edn. Addison Wesley, Reading, MA (1980)

2. Cassel, K.W.: Variational Methods with Applications in Science and Engineering. Cambridge University Press, New York (2013)

3. Gelfand, I.M., Fomin, S.V., Silverman, R.A. (eds.).: Calculus of variations (Unabridged repr. ed.). Dover Publications, Mineola, New York (2000)

4. Ferguson, J.: Brief survey of the history of the calculus of variations and its applications, available as arXiv:math/0402357 (2004)

5. Goldstine, H.H.: A History of the Calculus of Variations from the 17th Through the 19th Century. Springer, New York (2012)

6. Berdichevsky, V.L.: Variational Principles of Continuum Mechanics: I Fundamentals. Springer, Berlin, Heidelberg (2009)

7. Berdichevsky, V.L.: Variational Principles of Continuum Mechanics: II Applications. Springer, Berlin, Heildelberg (2009)

8. Abbasi, K.: Covid-19: politicisation, "corruption," and suppression of science, BMJ 371, m4425, available in https:// doi.org/10.1136/bmj.m4425 (2020)

9. Jankovic, I.: The political economy of mass panic, American Institute for Economic Research, available in https://www. aier.org/article/the-political-economy-of-mass-panic/ (2021)

10. Yates, C.: How to model a pandemic. The conversation, available in https://theconversation.com/ how-to-model-a-pandemic-134187 (2020)

11. Kermack, W.O., McKendrick, A.G.: A contribution to the mathematical theory of epidemics. Proc. R. Soc. A: Math. Phys. Eng. Sci. 115(772), 700 (1927) 
12. Wikipedia: Mathematical modelling of infectious disease, available in https://en.wikipedia.org/wiki/ (2020)

13. Wikipedia: Compartmental models in epidemiology, variations on the basic SIR model. available in https://en. wikipedia.org/wiki/ (2020)

14. Calafiore, G.C., Novara, C., Possieri, C.: A time-varying SIRD model for the COVID-19 contagion in Italy. Annu. Rev. Control 50, 361-372 (2020). https://doi.org/10.1016/j. arcontrol.2020.10.005

15. Chatterjee, S., Sarkar, A., Chatterjee, S., Karmakar, M., Paul, R.: Studying the progress of COVID-19 outbreak in India using SIRD model. Indian J. Phys. Proc. Indian Assoc. Cultiv. Sci. 2004, 1-17 (2020). https://doi.org/10. 1007/s12648-020-01766-8

16. Meunier, T.: Full lockdown policies in Western Europe countries have no evident impacts on the COVID-19 epidemic, available in https://www.medrxiv.org/content/10. 1101/2020.04.24.20078717v1 (2020)

17. Acemoglu, D., Chernozhukov, V., Werning, I., Whinston, M.D.: Optimal targeted lockdowns in a multi-group SIR model. National Bureau of Economic Research Working Paper 27102, available in http://www.nber.org/papers/ w27102 (2020)

18. Bendavid, E., Oh, C., Bhattacharya, J., Ioannidis, J.P.A.: Assessing mandatory stay-at-home and business closure effects on the spread of COVID-19. Eur. J. Clin. Invest., e13484. https://doi.org/10.1111/eci.13484 (2021)

19. Miltimore, J.: 3 studies that show lockdowns are ineffective at slowing COVID-19, Foundation for Economic Education, available at https://fee.org/articles/3-studies-that-show -lockdowns-are-ineffective-at-slowing - covid-19/, (2020)

20. American Institute for Economic Research, Lockdowns do not Control the Coronavirus: The evidence, available in https://www.aier.org/article/ lockdowns-do-not-control-the-coronavirus-the-evidence/ (2020)
21. Haug, N., Geyrhofer, L., Klimek, P. et al.: Ranking the effectiveness of worldwide COVID-19 government interventions. Nat. Hum. Behav. 4, 1303-1312, available in https:// www.nature.com/articles/s41562-020-01009-0 (2020)

22. Diffenbaugh, N.S., Field, C.B., Appel, E.A. et al.: The COVID-19 lockdowns: a window into the Earth System. Nat. Rev. Earth Environ. 1, 470- 481, available in https:// doi.org/10.1038/s43017-020-0079-1 (2020)

23. McKee, M., Stuckler, D.: If the world fails to protect the economy, COVID-19 will damage health not just now but also in the future. Nat. Med. 26, 640-642. https://doi.org/ 10.1038/s41591-020-0863-y (2020)

24. Haldane, A., Chowla, S.: Fast economic indicators. Nat. Rev. Phys. https://doi.org/10.1038/s42254-020-0236-y (2020)

25. American Institute for Economic Research, Cost of Lockdowns: A preliminary report, available in https://www.aier. org/article/cost-of-us-lockdowns-a-preliminary-report/ (2020)

26. Bertsekas, D.P.: Constrained Optimization and Lagrange Multiplier Methods. Academic Press, New York (1982)

27. Klein, D.: Lagrange multipliers without permanent scarring. Computer Science Division, University of California at Berkeley, available in https://people.eecs.berkeley.edu/ $\sim$ klein/papers/lagrange-multipliers.pdf (2020)

Publisher's Note Springer Nature remains neutral with regard to jurisdictional claims in published maps and institutional affiliations. 